\title{
Comparison of miRNA expression profiles in pituitary-adrenal axis between Beagle and Chinese Field dogs after chronic stress exposure
}

Wei Luo, Meixia Fang, Haiping Xu, Huijie Xing, Jiangnan Fu, Qinghua Nie

MicoRNAs (miRNAs), usually as gene regulators, participate in various biological processes, of which stress response is included. Hypothalamus-pituitary-adrenal axis (HPA axis) is an important pathway in regulating stress response. Although the mechanism that HPA axis regulates stress response has been basically revealed, the knowledge that miRNAs regulate stress response within HPA axis, still remains poor. The object of this study was to investigate the miRNAs in pituitary and adrenal cortex that regulated chronic stress response with high-throughput sequencing. The pituitary and adrenal cortex of Beagle and Chinese Field Dog (CFD) from a stress exposure group [including Beagle pituitary 1 (BP1), CFD pituitary 1 (CFDP1), Beagle adrenal cortex 1 (BAC1), CFD adrenal cortex 1 (CFDAC1)] and a control group [including Beagle pituitary 2 (BP2), CFD pituitary 2 (CFDP2), Beagle adrenal cortex 2 (BAC2), CFD adrenal cortex 2 (CFDAC2)], were selected for miRNA-seq comparisons. Comparisons, that were made in pituitary (including BP1 vs. BP2, CFDP1 vs. CFDP2, BP1 vs. CFDP1 and BP2 vs. CFDP2) and adrenal cortex (including BAC1 vs. BAC2, CFDAC1 vs. CFDAC2, BAC1 vs. CFDAC1 and BAC2 vs. CFDAC2), showed that a total of 39 and 18 common differentially expressed miRNAs (DE-miRNAs) (Total read counts $>1000$, Fold change $>2 \& P$-value $<0.001)$, that shared in at least two pituitary comparisons and at least two adrenal cortex comparisons, were detected separately. These identified DEmiRNAs were predicted for target genes, thus resulting in 3959 and 4010 target genes in pituitary and adrenal cortex, respectively. Further, 105 and 10 differentially expressed genes (DEGs) (Fold change $>2 \& P$-value $<0.05$ ) from those target genes in pituitary and adrenal cortex were obtained separately, in combination with our previous corresponding transcriptome study. Meanwhile, in line with that miRNAs usually negatively regulated their target genes and the dual luciferse reporter assay, we finally identified cfa-miR-205 might play an important role by up-regulating $M M D$ in pituitary and hippocampus, thus enhancing the immune response, under chronic stress exposure. Our results shed light on the miRNA expression profiles in pituitary and adrenal cortex with and without chronic stress exposure, and provide a new insight into miR-205 with its feasible role in regulating chronic stress in pituitary and hippocampus through targeting MMD. 
1 Comparison of miRNA expression profiles in pituitary-adrenal axis between Beagle and

\section{Chinese Field dogs after chronic stress exposure}

4 Wei Luo ${ }^{1,3 \#}$, Meixia Fang ${ }^{2 \#}$, Haiping $\mathrm{Xu}^{1,3}$, Huijie Xing ${ }^{2}$, Jiangnan $\mathrm{Fu}^{2 *}$, and Qinghua $\mathrm{Nie}^{1,3^{*}}$

$6{ }^{1}$ Department of Animal Genetics, Breeding and Reproduction, College of Animal Science, South

7 China Agricultural University, Guangzhou 510642, Guangdong, China.

8 2Institute of Laboratory Animals, Jinan University, Guangzhou, Guangdong 510632, Guangdong, 9 China.

$10{ }^{3}$ Guangdong Provincial Key Lab of Agro-Animal Genomics and Molecular Breeding and 11 National-Local Joint Engineering Research Center for Livestock Breeding, South China 12 Agricultural University \& Guangdong Wens Food Corporation, China.

13

14 \# Both authors contribute equally to this work.

15 *Both were corresponding authors: Jiangnan Fu (E-mail: fujiangnan126@126.com); Qinghua 16 Nie (nqinghua@scau.edu.cn)

17

18 Short title: the dog miRNA expression profile 


\section{Abstract}

20 MicoRNAs (miRNAs), usually as gene regulators, participate in various biological processes, of 21 which stress response is included. Hypothalamus-pituitary-adrenal axis (HPA axis) is an 22 important pathway in regulating stress response. Although the mechanism that HPA axis 23 regulates stress response has been basically revealed, the knowledge that miRNAs regulate stress 24 response within HPA axis, still remains poor. The object of this study was to investigate the 25 miRNAs in pituitary and adrenal cortex that regulated chronic stress response with high26 throughput sequencing. The pituitary and adrenal cortex of Beagle and Chinese Field Dog (CFD) 27 from a stress exposure group [including Beagle pituitary 1 (BP1), CFD pituitary 1 (CFDP1), 28 Beagle adrenal cortex 1 (BAC1), CFD adrenal cortex 1 (CFDAC1)] and a control group 29 [including Beagle pituitary 2 (BP2), CFD pituitary 2 (CFDP2), Beagle adrenal cortex 2 (BAC2), 30 CFD adrenal cortex 2 (CFDAC2)], were selected for miRNA-seq comparisons. Comparisons, 31 that were made in pituitary (including BP1 vs. BP2, CFDP1 vs. CFDP2, BP1 vs. CFDP1 and 32 BP2 vs. CFDP2) and adrenal cortex (including BAC1 vs. BAC2, CFDAC1 vs. CFDAC2, BAC1 vs. CFDAC1 and BAC2 vs. CFDAC2), showed that a total of 39 and 18 common differentially expressed miRNAs (DE-miRNAs) (Total read counts $>1000$, Fold change $>2 \& P$-value $<0.001$ ),

35 36

37 38 that shared in at least two pituitary comparisons and at least two adrenal cortex comparisons, were detected separately. These identified DE-miRNAs were predicted for target genes, thus resulting in 3959 and 4010 target genes in pituitary and adrenal cortex, respectively. Further, 105 and 10 differentially expressed genes (DEGs) (Fold change $>2 \& P$-value $<0.05$ ) from those target genes in pituitary and adrenal cortex were obtained separately, in combination with our previous corresponding transcriptome study. Meanwhile, in line with that miRNAs usually negatively regulated their target genes and the dual luciferse reporter assay, we finally identified cfa-miR-205 might play an important role by up-regulating $M M D$ in pituitary and hippocampus, thus enhancing the immune response, under chronic stress exposure. Our results shed light on the miRNA expression profiles in pituitary and adrenal cortex with and without chronic stress exposure, and provide a new insight into miR-205 with its feasible role in regulating chronic stress in pituitary and hippocampus through targeting $M M D$

Keywords dog, chronic stress exposure, HPA axis, miRNA-seq, cfa-miR-205 
49

50

51

52

53

54

55

56

57

58

59

60

61

62

63

64

65

66

67

68

69

\section{Introduction}

MiRNAs pertain to a class of non-coding endogenous RNAs with the lengths ranging from $18 \mathrm{nt}$ to $25 \mathrm{nt}$. They have been much explored as gene expression regulators that involve many biological processes, including differentiation, proliferation, development and apoptosis of cells, hormone secretions, virus diseases and cancers (Stefani \& Slark. 2008; Cordes et al. 2010; Taft et al. 2010). MiRNAs are also important in neuroendocrine system and functionally associated with in postembryonic development, axon guidance, synaptic plasticity and astrocyte activity (Bartel 2004; Krichevsky et al. 2006; Mor et al. 2011). Previous studies showed miR-18a could down-regulate the expression of glucocorticoid receptor in vitro (Uchida et al. 2008; Vreugdenhil et al. 2009). And in paraventricular nucleus, the miR-18a expression levels of the excessively stress-induced rats were higher than that in the natural rats. In addition, with the neuron treated by glucocorticoid (Kawashima et al. 2010), the miRNA-132 in neuron showed a lower expression level compared with that in negative control. Experiments in rats indicated that, compared with non-stress groups, the expression levels of some miRNAs in prefrontal cortex changed a bit within the acute stress exposure groups, but significantly up-regulated within the chronic stress exposure groups (Rinaldi et al. 2010). Collectively, the existing knowledge indicates that the miRNAs play important roles in neuroendocrine system and are closely associated with the stress response.

67 Hypothalamus-pituitary-adrenal axis (HPA axis) is an important part of the neuroendocrine system. Stress response, including acute and chronic ones, is regulated by the activity of the HPA axis in part (Frodl \& O’Keane 2013; Griffiths \& Hunter 2014). HPA axis is involved in maintaining the homeostasis of the body, with its regulations in digestion, immune system, mood, sex behavior, energy storage and consumption (Bodera et al. 2014; Kennedy et al. 2014; Gelman et al. 2015). Currently, the mechanism of HPA axis in regulating stress response has been basically revealed. However, especially in dog, the knowledge of the miRNAs associated with stress response in HPA axis is still devoid of a systemic revelation. In addition, to our knowledge, the systemic study of miRNAs in dog tissues still remains poor compared with that in other frequently-used medical animals, just with a study concerning dog trachea and dog lung reported (Zhao et al. 2014). In 2008, 357 candidate miRNAs in the Canis familiaris genome were identified with a comparative analysis of the whole genome in silico. Of them, 300 miRNAs were homological with characterized human miRNAs (Zhou et al. 2008). Virtually, miRNA is 
80 characterized by its high homology among species, thus the miRNA study targeting HPA axis in

81 dog can further its corresponding understanding of that in human or other animals as well.

82 High-throughput sequencing has been a powerful method to investigate the expression profiles 83 of miRNAs, and has been widely utilized in various organisms (Zhao et al. 2014; Zhan et al. 84 2014; Li et al. 2015; Wongwarangkana et al. 2015). In this study, we aimed to investigate the 85 miRNAs involving chronic stress response in dog pituitary and dog adrenal cortex (pituitary86 adrenal axis). To achieve it, the pituitary and adrenal cortex tissues of Beagles and Chinese Field 87 Dogs (CFD) that were treated with chronic stress exposure and non-treatment, were separately 88 performed with miRNA-seq. Then, in pituitary and adrenal cortex, miRNA profiles were 89 compared between the chronic stress exposure groups and the control groups within and between breeds to identify the miRNAs associated with chronic stress response in the pituitary-adrenal 91 axis.

92 To better pinpoint the pivotal miRNAs of regulating chronic stress in pituitary and adrenal 93 cortex, we combined the miRNA-seq of this study with our previous transcriptome study (Luo et 94 al. 2015.). Importantly, the animals used, stress exposure treatment, sampling and differential 95 expression analysis strategy in this study were all the same with our previous transcriptome study 96 (Luo et al. 2015.). In our transcriptome study, glucocorticoid levels of pre-and post-stress 97 exposure in each day were detected, and hippocampal sections of stress exposure groups and control groups in both breeds were conducted, both giving an evidence that our stress exposure was potent and valid. Besides, our previous transcriptome study identified a total of 511 and 171 differentially expressed genes in pituitary and adrenal cortex, respectively (Table S1).

101 The two dog breeds used here, including Beagle and CFD, own a distinct character in stress tolerance. Beagle is of good manageability, good environmental adaptability and good stress tolerance, while CFD is of excitability. We herein utilize two breeds that are distinct in stress tolerance but not just a single breed to avoid drawing a sweeping conclusion and pinpoint the miRNAs concerning chronic stress response more accurately. 
109 In this experiment, all animals used were approved by the Animal Care Committee of Jinan 110 University (Guangzhou, People's Republic of China) with approval number 20131018001, and 111 strictly implemented in line with the experimental basic principles.

\section{2.2 Chronic stress treatment}

113 Chronic stress treatment was given briefly as follows: 6 unrelated purebred male Chinese Field 114 Dogs (CFD) and 6 unrelated purebred male Beagles, similar in health, weight, and other aspects 115 were selected randomly. From each of these two breeds, three dogs were selected for stress 116 exposure via intermittent electrical stimulation and restraint stress, and the other 3 dogs of each

117 breed, used as normal control, were not exposed to the stress. Each morning for 10 days, dogs 118 were restrained and electrically stimulated with a stable current of $10 \mathrm{~mA}$ for $6 \mathrm{~s}$ followed by a 119 6-s interval without stimulation, lasting for 20 min every day. Meanwhile, before and after each 120 20-min stress exposure session, $4 \mathrm{ml}$ of blood was collected and isolated to examine cortisol 121 level with a cortisol radiation immunoassay kit used.

\subsection{Samples and RNA preparation}

123 All 12 dogs were killed by air embolism on the $11^{\text {th }}$ day, along with all parts of the adrenal 124 cortex and pituitary tissues collected and fast frozen in liquid nitrogen until for miRNA-seq. 125 Further, Trizol reagent (Invitrogen, USA) was used to isolate the RNAs of all the adrenal cortex 126 and pituitary tissues collected above. The quantity and quality of RNA were examined by 127 Agarose gel electrophoresis, NanoDrop 2000 (Thermo, USA), and Agilent 2100 (Agilent, USA). 128 The RNA collected from each group, including 3 dogs of each, were pooled with equal mass 129 respectively, thus obtaining 8 RNA pooled samples, including CFDP1 (CFD pituitary with stress 130 exposure), CFDP2 (CFD pituitary with non-disposal), CFDAC1 (CFD adrenal cortex with stress 131 exposure), CFDAC2 (CFD adrenal cortex with non-disposal), BP1 (Beagle pituitary with stress 132 exposure), BP2 (Beagle pituitary with non-disposal), BAC1 (Beagle adrenal cortex with stress 133 exposure), and BAC2 (Beagle adrenal cortex with non-disposal). In addition, tissues from the 134 hippocampus were collected to perform slice analysis (Nissl staining), aiming to evaluate the 135 influences of stress exposure on hippocampal region cells. 


\subsection{Small RNA library construction and sequencing}

137 A total of $1 \mu \mathrm{g}$ RNA was collected from each RNA pooled sample above. Then, specific 3' and 138 5' adaptors were added to the RNA ends of each group (Truseq TM Small RNA sample prep Kit, 139 Illumina). After that, the RNA with adaptors were reverse transcribed into its $1^{\text {st }} \mathrm{cDNA}$, with 140 random primers added. PCR was performed to amplify the cDNA with 11-12 cycles run and then 141 resulted in 8 cDNA libraries, including CFDP1, CFDP2, CFDAC1, CFDAC2, BP1, BP2, BAC1 142 and BAC2. After purified further by polyacrylamide gel electrophoresis (PAGE), each cDNA 143 library was performed by $1 * 50$ bp high-sequencing on Hiseq-2000 (Majorbio Inc, Shanghai, 144 China).

\subsection{Sequence data analysis}

146 The raw reads obtained by high-sequencing were further performed with quality control to result 147 in clean reads. In brief, the quality control was that of trimming low-quality reads (ambiguous $\mathrm{N}$ 148 and length < $18 \mathrm{nt}$ ), 3' adapters, 5' adapters and poly (A) sequences, with SeqPrep 149 (https://github.com/jstjohn/SeqPrep) and Sickle (https://github.com/najoshi/sickle) used. Thereby, 150 the lengths of 18-32 bp reads, as the clean ones, were obtained and calculated for their length 151 distributions, using Fastx-Toolkit (http://hannonlab.cshl.edu/fastx toolkit/). Meanwhile, the

152 identical reads were collapsed to obtain the unique reads for the statistics of the small RNA's 153 species and abundance. Moreover, the clean reads were mapped to Rfam database 154 (ftp://sanger.ac.uk/pub/databases/Rfam/) and GenBank noncoding RNA database 155 (http://blast.ncbi.nlm.nih.gov/) to annotate the miscellaneous RNAs, thus filtering out the rRNA, 156 scRNA, snoRNA, snRNA, tRNA and other non-coding RNA. The remaining sRNAs obtained 157 above, including known miRNAs and unknown miRNAs, were then mapped to the CanFam 3.1 158 genome to analyze their distributions on genome and calculate their expressive quantity. In 159 addition, the remaining sRNAs were mapped to the Canis Canine miRNA data of miRBase 21.0 160 to identify the known miRNAs. Meanwhile, the novel miRNAs were predicted using miRDeep2 161 (http://www.mdcberlin.de/en/research/research teams/systems biology of gene regulatory ele 162 ments/projects/miRDeep/index.html) from unannotated sRNAs (Bonnet et al. 2004; Langmead et 163 al. 2009; Friedländer et al. 2012). The data discussed here have been deposited in NCBI 164 Sequence Read Archive and is accessible through GEO Series accession no. GSE72015 165 (http://www.ncbi.nlm.nih.gov/geo/query/acc.cgi?acc=GSE72015). 
167 To explore the differentially expressed miRNAs (DE-miRNAs) in pituitary and adrenal, on the 168 basis of miRNA expression profiles, eight comparisons were made, including BP1_vs_BP2, 169 CFDP1_vs_CFDP2, BP1_vs_CFDP1 and BP2_vs_CFDP2 in pituitary; BAC1_vs_BAC2, 170 CFDAC1_vs_CFDAC2, BAC1_vs_CFDAC1 and BAC2_vs_CFDAC2 in adrenal cortex. Herein,

171 R package DEGseq using a Benjamini q-value of 0.001 as the cut-off (corrected p-value $<0.001$ ) 172 (http://www.bioconductor.org/packages/release/bioc/html/DEGseq.html) (Wang et al. 2010), 173 combined with Value1/Value2>2 (Value indicated the normalized count of samples), were used 174 to screen out the DE-miRNAs. Furthermore, the common miRNAs that differentially expressed 175 in at least two comparisons from pituitary and adrenal cortex were selected for the following 176 target prediction, respectively.

1772.7 Target gene prediction of DE-miRNA and its GO, KEGG analysis

178 For the target gene prediction, we herein utilized Targetscan 7.0 179 (http://www.targetscan.org/vert_70/). Importantly, target genes predicted with the cumulative 180 weighted context ++ score less than -0.2 , were selected for further analysis. Then, the target 181 genes were selected to perform GO and KEGG pathway analysis, with KOBAS 2.0 Functional 182 Annotation Tool (http://kobas.cbi.pku.edu.cn/program.inputForm.do?program=Annotate) 183 employed (Corrected $p$-value $<0.05$ ).

1842.8 Conjoint analysis of DE-miRNAs and DEGs

185 To better identify the important miRNAs in regulating chronic stress response, the DEGs 186 identified in our previous transcriptome study in pituitary and adrenal cortex were compared 187 with the target genes of DE-miRNAs in pituitary and adrenal cortex separately, thereby obtaining 188 the genes that were both DEGs and target genes in pituitary and adrenal cortex, respectively (as a 189 matter of convenience, the genes that were both DEGs and target genes were named "DE-target 190 genes"). Further, according to a negative correlation between miRNAs and their target genes in 191 expression pattern, the DE-miRNAs and their corresponding DE-target genes that against this 192 pattern, were then removed, thus resulting in the final DE-miRNA and its corresponding DE193 target gene [named DE-(miRNA-mRNA) pairs hereafter]. Subsequently, combined with the 194 expression level of DE-miRNAs and their corresponding DE-target genes' relatedness to stress 
195 regulation, a number of DE-(miRNA-mRNA) pairs were selected to perform dual luciferse 196 reporter assay.

1972.9 Validation of DE-miRNAs by $q P C R$

198 To elucidate the validity of the miRNA-seq data, DE-miRNAs, including miR-30a, miR-124, 199 miR-205 and miR-222, were further detected by qPCR.. In each group, $1 \mu \mathrm{g}$ of pooled RNA was 200 reverse transcribed using ReverTra Ace qPCR RT Kit 101 (TOYOBO, Japan), along with bulge201 loop RT primers added. The bulge-loop RT primers and primers for qPCR were both synthesized 202 by RIBOBIO (Guangzhou, China). QPCR was performed on the Bio-Rad S1000 with Bestar 203 SYBR Green RT-PCR Master Mix (DBI Bioscience, Germany). Besides, U6 and U48, as the 204 reference genes, were used to normalize the miRNA expression on the basis of $2^{-\Delta \Delta C T}$ method 205 (Schmittgen \& Livak 2008). The detailed information of the primers and the annealing 206 temperatures was presented in Table S2.

\section{3. Results}

\subsection{High-sequencing of small RNA}

210 In this study, eight libraries including BAC2, BAC1, CFDAC2, CFDAC1, BP2, BP1, CFDP2 211 and CFDP1 were constructed for miRNA-seq. As a result, a number of raw reads ranging from $21215,926,611$ to $81,028,035$ were obtained from these 8 groups (Table 1). After eliminating reads 213 of low quality (ambiguous $\mathrm{N}$ and length $<18 \mathrm{nt}$ ), $3^{\prime}$ adapters, $5^{\prime}$ adapters and poly (A) sequences, 214 a number of clean reads ranging from 11,900,933 to 74,445,014 were obtained from the 8 groups 215 (Table 1). Further, the clean reads from each group were mapped to Rfam database (11.0, 216 http://Rfam.sanger.ac.uk/) and GenBank noncoding RNA database (http://blast.ncbi.nlm.nih.gov/) 217 to annotate miscellaneous RNAs. And the detailed annotations of small RNA in each library 218 were presented in Figure S1.

219 The statistics of size distributions showed that the majority of sRNAs were 21-23 nt in length 220 across 8 samples, along with a significantly down-regulated profile in post-stress group 221 compared with that in pre-stress (Figure 1A and 1B). Besides, when mapped to the reference 222 genome of Canis Canine with the remaining sRNA reads (including known miRNAs and 223 unknown miRNAs), Chromosome 1, 3, 5, 20 and 31 were found to be mapped most abundantly 
224 (ratio $>5 \%$ ) in dog (Figure 2). Meanwhile, a bigger number of small RNA reads were showed in 225 pituitary samples (especially in CFD pituitary samples) compared with that in adrenal cortex 226 samples.

\subsection{Identification of known miRNAs}

228 When mapped to the Canis Canine data of miRBase 21.0 by the remaining sRNA reads, a total 229 of 425 out of 453 known miRNAs (Table S3) were identified across eight samples. Of the 425 230 identified known miRNAs, BAC2, BAC1, CFDAC2, CFDAC1, BP2, BP1, CFDP2 and CFDP1 231 contributed to 332, 325, 332, 345, 327, 318, 352 and 355 miRNAs, respectively.

232 Besides, the importance attached to the abundance of the miRNA expression in these two 233 breeds was conducive to identify the miRNAs of high activity in dog pituitary and dog adrenal 234 cortex. In adrenal cortex samples, cfa-miR-99a was the most abundant known miRNA, followed 235 with cfa-miR-30 family (including cfa-miR-30a, cfa-miR-30b, cfa-miR-30c and cfa-miR-30d), 236 cfa-miR-26 family (including cfa-miR-26a and cfa-miR-26b) and cfa-miR-7 family (including 237 cfa-miR-7 and cfa-miR-7g) (The top 20 known miRNAs of abundance in adrenal cortex were 238 listed in Table S4). Meanwhile, cfa-miR-7 family (including cfa-miR-7 and cfa-miR-7g), cfa239 miR-99a, cfa-miR-30 family (including cfa-miR-30a and cfa-miR-30d), and cfa-miR-125 family 240 (including cfa-miR-125a and cfa-miR-125b) were found to be the most abundant known 241 miRNAs in pituitary samples (The top 20 known miRNAs of abundance in pituitary were listed 242 in Table S4). In previous high-throughput sequencing studies of other species, various isoforms 243 of the miRNAs were always detected (Li et al. 2011; Visser et al. 2014). In this study, we found 244 almost all known cfa-miRNAs were of isoforms, with a larger number of isoforms possessed by 245 the miRNAs of higher abundance. Moreover, most of the isoforms showing the highest 246 expression level were identical to the canonical forms in miRBase 20.0, but for some other 247 miRNAs, like the cfa-miR-217, the isoforms that showed the highest expression level were not 248 the canonical ones at all (Figure S2), indicating the variability of miRNA expression pattern.

\subsection{Identification of novel miRNAs}

250 In this study, a total of 147 novel miRNAs were identified across eight samples (Table S5).

251 Compared with the known miRNAs identified here, rather lower expression levels were showed 252 by the identified novel miRNAs. Of the 147 novel miRNAs, there were just 18 ones that were 253 greater than 1000 reads in expression level (Table S6). MiR-24_20231 and miR-21_17074 as the 
254 top two of the 18 novel miRNAs above, their predicted secondary structures were presented in

255 Figure S3. Besides, of the 147 novel miRNAs, 75 ones shared a same seed sequence with some

256 other known miRNAs of other species (especially mammals).

\subsection{Identification of differentially expressed known miRNAs}

258 In this study, a total of 27 (6 up, 21 down), 37 (18 up, 19 down), 30 (22 up, 8 down), 21 (15 up, 6

259 down), 25 (19 up, 6 down), 58 (13 up, 45 down), 32 (7 up, 25 down) and 43 (32 up, 11 down) 260 DE-miRNAs $\left(P\right.$-value $<0.001, \log _{2}($ Fold_change $)>1$ ) were detected in the comparisons of 261 BAC1_vs_BAC2, BAC1_vs_CFDAC1， BAC2_vs_CFDAC2， CFDAC1_vs_CFDAC2, 262 BP1_vs_BP2, BP1_vs_CFDP1, BP2_vs_CFDP2 and CFDP1_vs_CFDP2 respectively (the DE263 miRNAs identified from adrenal cortex were listed in Table S7, and that from pituitary were 264 listed in Table S8). Furthermore, a total of 90 miRNAs expressing differentially in pituitary 265 comparisons were obtained, with 51 common DE-miRNAs shared in at least 2 pituitary 266 comparisons (common DE-miRNAs). Of the 51 common DE-miRNAs, cfa-miR-105a, cfa-miR267 219-3p and cfa-miR-802 were the common DE-miRNAs of the four pituitary comparisons. 268 Meanwhile, of the 62 DE-miRNAs detected in adrenal cortex comparisons, there were 39 269 common DE-miRNAs, with the three common miRNAs of cfa-miR-196a, cfa-miR-216b, and 270 cfa-miR-514 shared in all these four adrenal cortex comparisons. In consideration of the 271 functional impact exerted by miRNA expression level, we herein chose the DE-miRNAs that 272 whose total reads within pituitary or adrenal cortex were greater than 1000 for subsequent 273 analysis, thus resulting in a total of 39 and 18 common DE-miRNAs in pituitary and adrenal 274 cortex separately (Figure 3; Table 3).

275 To validate the miRNA expression levels obtained by high-sequencing, 4 random DE276 miRNAs, including cfa-miR-30a, cfa-miR-124, cfa-miR-205 and cfa-miR-222 were selected to 277 perform qPCR (Figure 4). The results showed cfa-miR-30a, cfa-miR-205 both were significantly 278 down-regulated in CFDP1_vs_CFDP2 [| $\log _{2}$ (fold change) $\left.\mid>1\right]$. Cfa-miR-124 was significantly 279 up-regulated but not significantly for cfa-miR-222 in CFDP1_vs_CFDP2 [|Log 2 (fold change) $\mid>1]$. 280 These observations were basically consistent with those obtained by high-sequencing, indicating 281 the high-throughput sequencing data was reliable. 
283 The common DE-miRNAs, including 33 and 22 in pituitary and adrenal cortex were used for 284 miRNA target prediction, with the methods of Targetscan used. Consequently, 3959 and 4010 285 target genes were obtained from pituitary and adrenal cortex respectively (Table S9). Then 3959 286 and 4010 target genes were performed with GO and KEGG analysis separately. Results showed 287 that neither of the target genes from pituitary and adrenal cortex could be clustered into a certain 288 GO term or KEGG pathway, due to the corrected p-value $>0.05$. Instead, IPA was employed to 289 identify the canonical pathways enriched by the predicted target genes. In CFDP1_vs_CFDP2 290 (i.e., 3959 predicted target genes), the most enriched pathway was the axon guidance signaling 291 pathway $(\mathrm{p}=1.34 \mathrm{E}-11)$.

292

293 294

295

296

297

298

299

300

301

302

303

304

305

306

307

308

309

310

311

312

\subsection{DE-(miRNA-mRNA) pairs identification}

In our previous transcriptome study (Luo et al. 2015.), a total of 511 and 171 DEGs were identified in pituitary and adrenal cortex, respectively (Table S1). The DEGs identified in our transcriptome study were separately compared with the target genes in pituitary and adrenal cortex (i.e., 3959 and 4010 respectively), thus resulting in 105 and 10 common genes that were both included in DEGs and target genes (DE-target genes), respectively. Furthermore, the expression patterns of the 105 and 10 DE-target genes were compared with that of their corresponding miRNAs, without prejudice to the negative correlations between miRNAs and their target genes in both breeds. Consequently, 18 DE-(miRNA-mRNA) pairs in pituitary were obtained (Table 3). In consideration of the miRNA expression level and its potential target genes' relatedness to stress regulation, we here selected 6 DE-(miRNA-mRNA) pairs to perform dual luciferase reporter assay to elucidate their dependability, including cfa-miR-30a-GRIA2, cfa-miR-30a-SORCS3, cfa-miR-30a-SLC1A2, cfa-miR-205-MMD, cfa-miR-205-GLRB and cfamiR-205-CHN1. The dual luciferase reporter assay results showed just the cfa-miR-205-MMD conformed with the predicted (Figure 5A), suggesting an important role played by cfa-miR-205 in regulating $M M D$ under stress exposure in pituitary. Further, $M M D$ and cfa-miR-205 expression levels were examined in CFD brain, cerebellum, hippocampus and hypothalamus tissues by qPCR, normalized with $G A P D H, U 6$ and $U 48$ respectively. Expectedly, $M M D$ was found to be upregulated, while down-regulated for cfa-miR-205 in CFDP1_vs_CFDP2 (Figure $5 B)$. 


\section{4. Discussion}

314 Dog, especially Beagle, as a typical medical experimental animal has been utilized prevalently 315 (Lu et al. 2015; Ji et al. 2015; Li et al. 2015). In this study, we took advantage of the good 316 manageability and good stress tolerance in Beagle, and the excitability in CFD, to explore the

317 differentially expressed miRNAs in pituitary-adrenal axis under chronic stress exposure within 318 and between breeds, with miRNA-seq. Our miRNA-seq study yielded 28,667,214, 15,926,611, $31932,583,375,32,665,593,27,591,701,18,359,529,81,028,035$, and 51,202,889 raw reads in 320 BAC2, BAC1, CFDAC2, CFDAC1, BP2, BP1, CFDP2 and CFDP1, respectively. To our 321 knowledge, this is the first time that the miRNA profiles of dog pituitary and dog adrenal cortex 322 were presented. We found cfa-miR-30 family (including cfa-miR-30a and cfa-miR-30d), cfa323 miR-7 family (including cfa-miR-7 and cfa-miR-7g) and cfa-miR-125 (including cfa-miR-125a 324 and cfa-miR-125b) were the miRNAs of highest activity in dog pituitary. Besides, cfa-miR-99a, 325 cfa-miR-30 family (including cfa-miR-30a and cfa-miR-30d), cfa-miR-26 family (including cfa326 miR-26a and cfa-miR-26b) and cfa-miR-7 family (including cfa-miR-7 and cfa-miR-7g) were 327 the miRNAs of highest activity in dog adrenal cortex. These observations in pituitary and adrenal 328 cortex were distinct from those in dog lung and dog trachea, in which the cfa-miRNA-143 and 329 the cfa-let-7 were the ones of highest activity (Zhao et al. 2014) respectively. Furthermore, when 330 we compared the miRNA expression profiles of pre-and post- stress exposure groups with that of 331 their corresponding gene expression profiles, especially in pituitary, a strikingly converse 332 expression pattern was observed between the miRNA expression profiles and gene expression 333 profiles, indirectly proving that the negatively regulated pattern between miRNAs and genes 334 existed. Intriguingly, in non-disposal groups, within both breeds, the total miRNA expression 335 levels of the pituitary were significantly higher than that of the adrenal cortex (The total reads of 336 21, 22, and $23 \mathrm{nt}$ detected in CFD pituitary were all greater than 15 million, while all less than 8 337 million in CFD adrenal cortex, and this phenomenon was also found in Beagle but not so 338 significantly compared with that in CFD). Apart from the deviation caused by high-sequencing, 339 this might be attributable to the wider roles played in biological functions by pituitary than that 340 by adrenal cortex, as a larger miRNA reserve pool could irrigate more. Besides, in non-disposal 341 groups, CFD pituitary exhibited a much higher miRNA expression level than that of Beagle 342 pituitary. This might in part explain the different stress tolerance between Beagle and CFD:

343 Because the expression pattern of the miRNA was negatively correlated with its target genes, 
344 and the genes involving chronic stress response in pituitary were mainly up-regulated, hence a

345 larger miRNA reserve pool harbored by CFD pituitary indicated that the CFD had greater 346 potential energy to up-regulate the genes involving stress response to a greater degree, thus 347 expressing symptoms more severely in CFD.

348 Because the miRNAs function as gene regulators, it's of limited significance to just discuss 349 the roles of miRNAs without concerning their target gene expressions. In this study, we 350 combined the miRNA expression profiles with their corresponding transcriptome profiles to

351

352 353

354

355

356

357

358

359

360

361

362

363

364

365

366

367

368 369

370

371

372 373 analyze the potential DE-(miRNAs-target genes). Combined with the expression level and the relatedness to stress regulation, six DE-(miRNA-mRNA) pairs, including cfa-miR-30a-GRIA2, cfa-miR-30a-SORCS3, cfa-miR-30a-SLC1A2, cfa-miR-205-MMD, cfa-miR-205-GLRB and cfamiR-205-CHN1 were identified as the pivotal candidate miRNAs and their corresponding target genes. The further dual luciferase reporter assay indicated a significant target relation between cfa-miR-205 and MMD. We herein just identified one pivotal DE-(miRNA-mRNA) pair, and this could be partly accounted for our rigorous strategy of the statistical analysis and the control group set, thus inducing some true positive DE-(miRNA-mRNA) pairs being omitted. More importantly, these results strongly indicated an actual positive correlated expression pattern between most miRNAs and their corresponding genes under chronic stress exposure, indicating a general buffering role for miRNAs in gene expression but not a role of synergy. In this study, miRNAs that were differentially expressed in at least two comparisons within pituitary or adrenal cortex groups were regarded as the candidate miRNAs for target prediction, and the target genes expressing differentially were selected. In addition, we herein didn't take the miRNA and its corresponding target genes into consideration if the expression pattern between the miRNA and its target genes was just negatively correlated in one breed but not in another. These measures all contributed to a smaller number of the candidate DE-miRNAs and their corresponding target genes, but helped a lot in identifying the miRNAs of major effect on chronic stress regulation..

In this study, through conjoint analysis of mRNA-seq and miRNA-seq studies, we identified cfa-miR-205 might play a important role in regulating $M M D$ in pituitary under chronic stress exposure. $M M D$ (monocyte to macrophage differentiated-associated) has been found to be upregulated upon monocyte differentiation (Liu et al., 2012). In our previous hippocampal sections 
374 upon the CFD and the Beagle with and without chronic stress exposure, we found the CFD

375 hippocampus underwent stress exposure exhibited the largest apoptosis compared with other 376 groups (Luo et al., 2015), suggesting a potential immune response in it. Thus, in

377 CFDP1_vs_CFDP2, we hypothesized MMD should be up-regulated, along with the cfa-miR-205

378 be down-regulated, as more macrophages were transferred from monocytes as supposed.

379 Inspiringly, the qPCR results supported our hypothesis (Figure 5B), indicating a potential pivotal

380 role for cfa-miR-205 in regulating $M M D$ under chronic stress exposure.

\section{Conclusions}

382 In conclusion, we herein compared the miRNA expression profiles of the adrenal cortex and 383 pituitary in Beagle and CFD with and without stress exposure. We detected 425 known miRNAs 384 and 147 novel miRNAs in total across 8 samples, and of the 425 known miRNAs, a total of 90 385 and 62 miRNAs were found to be differentially expressed in pituitary and adrenal cortex 386 respectively. In combination with the transcriptome profiles corresponding to the miRNA 387 profiles of this study, we identified cfa-miR-205 might play an important role by up-regulating $388 M M D$, thus enhancing the immune response in pituitary and hippocampus, under chronic stress 389 exposure.. Our results shed light on the miRNA expression profiles in pituitary and adrenal 390 cortex with and without chronic stress exposure, and provide a new insight into miR-205 with its 391 feasible role in regulating chronic stress in pituitary and hippocampus through targeting $M M D$.

392

393 Competing interests

394 The authors have declared that no competing interests exist.

395 Authors' contributions

396 WL and MF carried out the molecular genetic studies, participated in the sequence alignment, 397 statistical analysis, and drafted the manuscript. HPX reviewed drafts of the paper. MF and HJX 
398 contributed reagents/materials/analysis tools. QN and FJ conceived and designed the study. All

399 authors read and approved the final manuscript.

\section{Acknowledgements}

401 The funders played no role in the study design, data collection and analysis, the decision to 402 publish, or in the preparation of the manuscript. 


\section{References}

404 1. Bartel DP. 2004. MicroRNAs: genomics, biogenesis, mechanism, and function. Cell $405 \quad 116: 281-297$.

406 2. Bodera P, Stankiewicz W, Kocik J. 2014. Interactions of orphanin FQ/nociceptin (OFQ/N)

407 system with immune system factors and hypothalamic-pituitary-adrenal (HPA) axis. Pharmacological Reports 66(2):288-91. DOI: 10.1016/j.pharep.2013.12.003.

409

410

411

412

3. Bonnet E, Wuyts J, Rouzé P, Van de Peer Y. 2004. Evidence that microRNA precursors, unlike other non-coding RNAs, have lower folding free energies than random sequences. Bioinformatics 20:2911-7.

4. Cordes KR, Srivastava D, Ivey KN. 2010. MicroRNAs in Cardiac Development. Pediatric Cardiology 31(3):349-56. DOI: 10.1242/dev.052647.

5. Friedländer MR, Mackowiak SD, Li N, Chen W, Rajewsky N. 2012. miRDeep2 accurately identifies known and hundreds of novel microRNA genes in seven animal clades. Nucleic Acids Research 40:37-52. DOI: 10.1093/nar/gkr688.

6. Frodl T. \& O'Keane V. (2013) How does the brain deal withcumulative stress? A review with focus on developmental stress, HPA axis function and hippocampal structure in humans.Neurobiology of Disease 52, 24-37.

7. Gelman PL, Flores-Ramos M, López-Martínez M, Fuentes CC, Grajeda JP. 2015. Hypothalamic-pituitary-adrenal axis function during perinatal depression. Neuroscience Bull 31(3):338-50. DOI: 10.1007/s12264-014-1508-2.

8. Griffiths B.B. \& Hunter R.G. (2014) Neuroepigenetics of stress. Neuroscience 275, 420-35.

9. Ji X, Bao N, An KN, Amadio PC, Steinmann SP, Zhao C. 2015. A Canine Non-WeightBearing Model with Radial Neurectomy for Rotator Cuff Repair. PLoS One 10(6):e0130576.

10. Kawashima H, Numakawa T, Kumamaru E, Adachi N, Mizuno H, Ninomiya M, Kunugi H, Hashido K. 2010. Glucocorticoid attenuates brain-derived neurotrophic factor-dependent 
428

429

430

431

432

433

434

435

436

437

438

439

440

441

442

443

444

445

446

447

448

449

450

451

452

upregulation of glutamate receptors via the suppression of microRNA-132 expression. Neuroscience 165(4):1301-11. DOI: 10.1016/j.neuroscience.2009.11.057.

11. Kennedy PJ, Cryan JF, Quigley EM, Dinan TG, Clarke G. 2014. A sustained hypothalamicpituitary-adrenal axis response to acute psychosocial stress in irritable bowel syndrome. Psychological Medicine 44(14):3123-34. DOI: 10.1017/S003329171400052X.

12. Krichevsky AM, SonnEffective KC, Isacson O, Kosik KS. 2006. Specific microRNAs modulate embryonic stem cell-derived neurogenesis. Stem Cells 24:857-864.

13. Langmead B, Trapnell C, Pop M, Salzberg SL. 2009. Ultrafast and memory-efficient alignment of short DNA sequences to the human genome. Genome Biology 10(3):R25. DOI: 10.1186/gb-2009-10-3-r25.

14. Li T, Wu R, Zhang Y, Zhu D. 2011. A systematic analysis of the skeletal muscle miRNA transcriptomee of chicken varieties with divergent skeletal muscle growth identifies novel miRNAs and differentially expressed miRNAs. BMC Genomics 12:186. DOI: 10.1186/1471-2164-12-186.

15. Li W, Yan S, Zhao J, Ding X, Zhang S, Wang D, Liu L, Peng W, Li H, Wang D, Liu Z, Li Y. 2015. Metoprolol Inhibits Cardiac Apoptosis and Fibrosis in a Canine Model of Chronic Obstructive Sleep Apnea. Cell Physiology and Biochemistry 36(3):1131-1141.

16. Li Z, Chen B, Feng M, Ouyang H, Zheng M, Ye Q, Nie Q, Zhang X. 2015. MicroRNA-23b Promotes Avian Leukosis Virus Subgroup J (ALV-J) Replication by Targeting IRF1. Scientific Reports. 18;5:10294. DOI: 10.1038/srep10294.

17. Lu J, Wang Z, Zhou T, Chen S, Chen W, Du H, Tan Z, Yang H, Hu X, Liu C, Ling Z, Liu Z, Zrenner B, Woo K, Yin Y. 2015. Selective Proximal Renal Denervation Guided by Autonomic Responses Evoked via High-Frequency Stimulation in a Preclinical Canine Model. Circulation-Cardiovascular

Interventions $8(6)$.

DOI: 10.1161/CIRCINTERVENTIONS. 
453 18. Luo W, Fang MX, Xu HP, Xing HJ, Nie QH. 2015. Transcriptomee comparison in the 454 pituitary-adrenal axis between Beagle and Chinese Field dogs after chronic stress exposure. Animal Genetics. Article first published online: 7 JUL 2015. DOI: 10.1111/age.12325.

456

457

458

459

460

461

462

463

464

465

466

467

468

469

470

471

472 473

474

475

476

477

478

19. Mor E, Cabilly Y, Goldshmit Y, Zalts H, Modai S, Edry L, Elroy-Stein O, Shomron N. 2011. Species-specific microRNA roles elucidated following astrocyte activation. Nucleic Acids Research 39(9):3710-23. DOI: 10.1093/nar/gkq1325.

20. Rinaldi A, Vincenti S, De Vito F, Bozzoni I, Oliverio A, Presutti C, Fragapane P, Mele A. 2010. Stress induces region specific alterations in microRNAs expression in mice. Behavioral Brain Research 208(1):265-269. DOI: 10.1016/j.bbr.2009.11.012.

21. Schmittgen, TD \& Livak KJ. 2008. Analyzing real-time PCR data by the comparative C-T method. Nature Protocols 3(6):1101-8.

22. Stefani G, Slark FJ. 2008. Small non-coding RNAs in animal development. Nature Reviews Molecular Cell Biology 9(3):219-30. DOI: 10.1038/nrm2347.

23. Taft RJ, Pang KC, Mercer TR, Dinger M, Mattick JS. 2010. Non-coding RNAs: regulators of disease. Journal of Pathology 220(2):126-39. DOI: 10.1002/path.2638.

24. Uchida S, Nishida A, Hara K, Kamemoto T, Suetsugi M, Fujimoto M. 2008. Characterization of the vulnerability to repeated stress in Fischer 344 rats: possible involvement of microRNA-mediated down-regulation of the glucocorticoid receptor. European Journal of Neuroscience 27:2250-2261. DOI: 10.1111/j.1460-9568.2008.06218.x.

25. Visser M, van der Walt AP, Maree HJ, Rees DJ, Burger JT. 2014. Extending the sRNAome of apple by next-generation sequencing. PLoS One 9(4):e95782. DOI: 10.1371/journal.pone.0095782.

26. Vreugdenhil E, Verissimo CS, Mariman R, Kamphorst JT, Barbosa JS, Zweers T, Champagne DL, Schouten T, Meijer OC, de Kloet ER, Fitzsimons CP. 2009. MicroRNA 18 and 124a down-regulate the glucocorticoid receptor: implications for glucocorticoid responsiveness in the brain. Endocrinology 150: 2220-2228. doi: 10.1210/en.2008-1335. 
479 27. Wang L, Feng Z, Wang X, Wang X, Zhang X. 2010. DEGseq: an R package for identifying

480

481

482

483

484

485

486

487

488

489

490

491

492

493

494

495

496

497 differentially expressed genes from RNA-seq data. Bioinformatics 26:136-8. DOI: 10.1093/bioinformatics/btp612.

28. Wongwarangkana C, Fujimori KE, Akiba M, Kinoshita S, Teruya M, Nezuo M, Masatoshi T, Watabe S, Asakawa S. 2015. Deep sequencing, profiling and detailed annotation of microRNAs in Takifugu rubripes. BMC Genomics. 16:457.

29. Zhan C, Yan L, Wang L, Jiang W, Zhang Y, Xi J, Chen L, Jin Y, Qiao Y, Shi Y, Wang Q. 2014. Identification of reference miRNAs in human tumors by TCGA miRNA-seq data. Biochemical and Biophysical Research Communications 453(3):375-8.

30. Zhao FR, Su S, Zhou DH, Zhou P, Xu TC, Zhang LQ, Cao N, Qi WB, Zhang GH, Li SJ. 2014. Comparative analysis of microRNAs from the lungs and trachea of dogs (Canis familiaris) infected with canine influenza virus. Infect Genetic Evolution 21:367-74.

31. Zhou D, Li S, Wen J, Gong X, Xu L, Luo Y. Genome-wide computational analyses of microRNAs and their targets from Canis familiaris. Computational Biology and Chemistry. 2008, 32(1): 60-6.

32. Liu Q, Zheng J, Yin DD, Xiang J, He F, Wang YC, Liang L, Qin HY, Liu L, Liang YM, Han H. Monocyte to macrophage differentiation-associated (MMD) positively regulates ERK and Akt activation and TNF- $\alpha$ and NO production in macrophages. Mol Biol Rep. 2012 , 39(5):5643-5 


\section{Table $\mathbf{1}$ (on next page)}

Statistics of miRNA-seq across 8 samples in brief.

$\mathrm{BAC1}$, Beagle adrenal cortex with treatment; BAC2, Beagle adrenal cortex without treatment; BP1, Beagle pituitary with treatment; BP2, Beagle pituitary without treatment; CFDAC1, Chinese Field Dog adrenal cortex with treatment; CFDAC2, Chinese Field Dog adrenal cortex without treatment; CFDP1, Chinese Field Dog pituitary with treatment; CFDP2, Chinese Field Dog pituitary without treatment. 


\section{Table 1. Statistics of miRNA-seq across 8 samples in brief.}

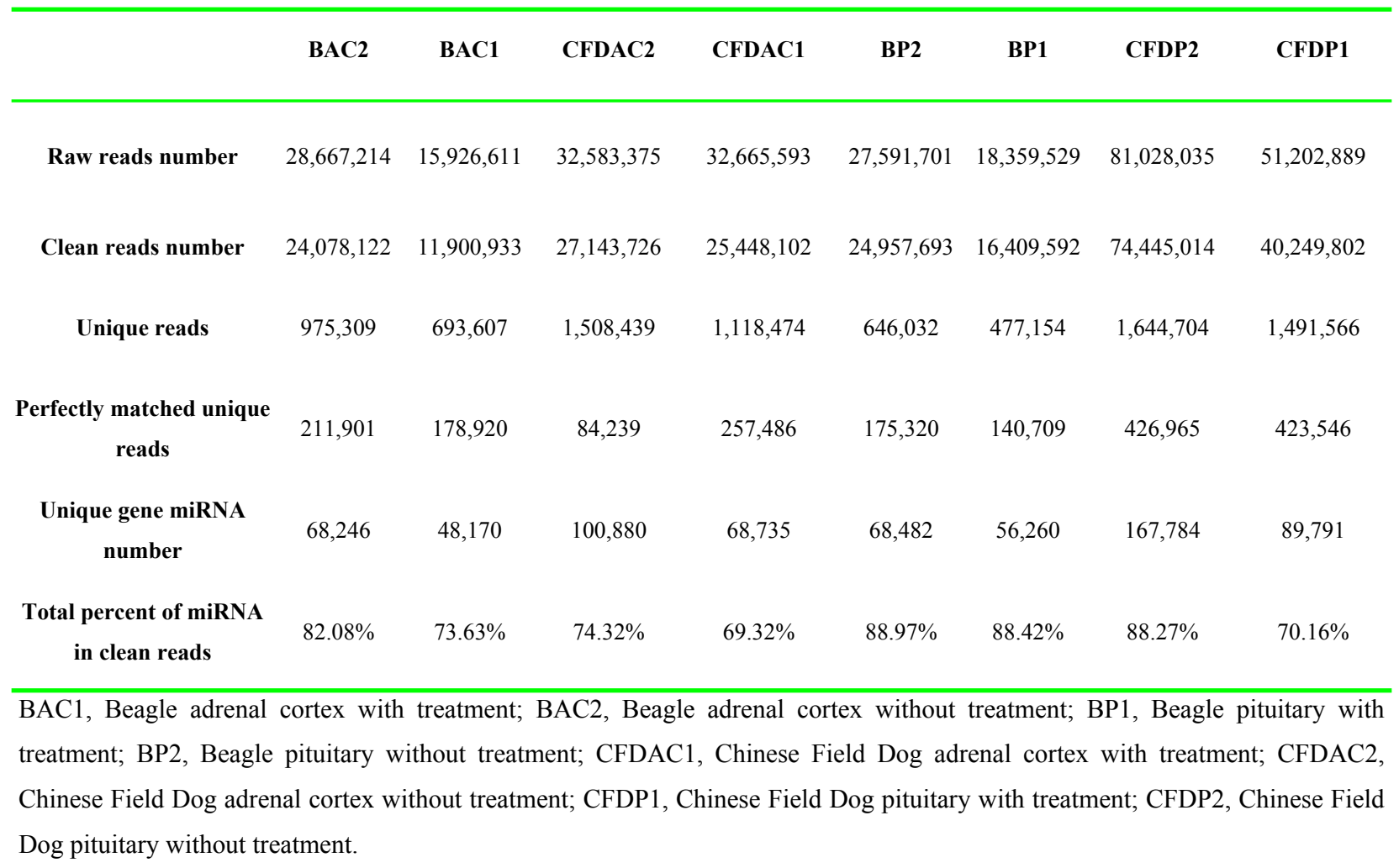




\section{Table 2 (on next page)}

39 and 18 common differentially expressed miRNAs pituitary from pituitary and adrenal cortex respectively. 
1 Table 2. 39 and 18 common differentially expressed miRNAs pituitary from pituitary and

2 adrenal cortex respectively.

\begin{tabular}{|c|c|c|c|}
\hline \multirow{2}{*}{ miRNA ID } & \multicolumn{2}{|c|}{ Normalized Reads } & \multirow{2}{*}{$\mid \log 2($ Fold_change)| } \\
\hline & Sample 1 & Sample 2 & \\
\hline \multirow{2}{*}{ cfa-miR-30a } & CFDP1:2553208 & CFDP2:5289957 & 1.05 \\
\hline & BP1:6285675 & CFDP1:2553208 & 1.3 \\
\hline \multirow{2}{*}{ cfa-miR-9 } & CFDP $1: 2270770$ & CFDP2:548039 & 2.05 \\
\hline & BP $1: 788518$ & CFDP1: 2270770 & 1.53 \\
\hline & CFDP 1:1806445 & CFDP2:67009 & 4.75 \\
\hline \multirow[t]{2}{*}{ cfa-miR-124 } & BP1:51801 & CFDP1:1806445 & 5.12 \\
\hline & BP2:26408 & ТP2:67009 & 1.34 \\
\hline \multirow[t]{2}{*}{ cfa-miR-144 } & $\begin{array}{l}\text { CFDP1: } \\
162116\end{array}$ & CFDP2: 341015 & 1.07 \\
\hline & BP1: 561345 & CFDP1: 162116 & 1.79 \\
\hline cfa-miR-128 & $\begin{array}{l}\text { CFDP1: } \\
439481\end{array}$ & CFDP2:86512 & 2.34 \\
\hline \multirow{3}{*}{ cfa-miR-146b } & BP1: 105386 & TP1: 439481 & 2.06 \\
\hline & BP1: 109353 & BP2: 51299 & 1.09 \\
\hline & BP2: 51299 & BP1: 163222 & 1.67 \\
\hline \multirow[t]{2}{*}{ cfa-miR-222 } & $\begin{array}{l}\text { CFDP1: } \\
153482\end{array}$ & CFDP2: 29889 & 2.36 \\
\hline & BP1: 17161 & CFDP1: 153482 & 3.16 \\
\hline \multirow{2}{*}{ cfa-miR-34c } & BP1: 53532 & CFDP1: 6620 & 3.02 \\
\hline & BP2: 77871 & BP1: 12722 & 2.61 \\
\hline \multirow{2}{*}{ cfa-miR-221 } & CFDP1: 90710 & CFDP2: 19204 & 2.24 \\
\hline & BP1: 10704 & CFDP1: 90710 & 3.08 \\
\hline \multirow{3}{*}{ cfa-miR-95 } & BP1: 23657 & CFDP1: 47878 & 1.02 \\
\hline & BP2: 13824 & CFDP2: 31697 & 1.2 \\
\hline & BP1: 10730 & BP2: 22724 & 1.08 \\
\hline \multirow[t]{2}{*}{ cfa-miR-205 } & CFDP1: 12765 & CFDP2: 55847 & 2.13 \\
\hline & BP2: 22724 & CFDP2: 55847 & 1.3 \\
\hline \multirow{2}{*}{ cfa-miR-138a } & CFDP1: 52944 & CFDP2: 7943 & 2.74 \\
\hline & BP1: 10264 & CFDP1: 52944 & 2.37 \\
\hline \multirow{2}{*}{ cfa-miR-135b } & CFDP $1: 10065$ & CFDP2:21001 & 1.06 \\
\hline & BP1:28943 & CFDP1:10065 & 1.52 \\
\hline \multirow{4}{*}{$\begin{array}{l}\text { cfa-miR-155 } \\
\text { cfa-miR-885 }\end{array}$} & BP1:12914 & CFDP1:26499 & 1.04 \\
\hline & BP2:7255 & CFDP2:14735 & 1.02 \\
\hline & CFDP $1: 22660$ & CFDP2:9447 & 1.26 \\
\hline & BP1:7043 & CFDP1:22660 & 1.69 \\
\hline
\end{tabular}




\begin{tabular}{|c|c|c|c|}
\hline \multirow{4}{*}{$\begin{array}{l}\text { cfa-miR-150 } \\
\text { cfa-miR-326 }\end{array}$} & CFDP1:17024 & CFDP2:4522 & 1.91 \\
\hline & BP1:3981 & CFDP1:17024 & 2.1 \\
\hline & CFDP1:13307 & CFDP2:4126 & 1.69 \\
\hline & $\mathrm{BP} 1: 5618$ & CFDP1:13307 & 1.24 \\
\hline \multirow{3}{*}{ cfa-miR-105a } & $\mathrm{BP} 1: 2223$ & BP2:1031 & 1.11 \\
\hline & CFDP1:16712 & CFDP2:3167 & 2.4 \\
\hline & $\mathrm{BP} 1: 2223$ & CFDP1:16712 & 2.91 \\
\hline \multirow{4}{*}{$\begin{array}{l}--------- \\
\text { cfa-miR-330 }\end{array}$} & BP2:1031 & CFDP2:3167 & 1.62 \\
\hline & CFDP 1:9455 & CFDP2:3793 & 1.32 \\
\hline & BP1:3954 & CFDP1:9455 & 1.26 \\
\hline & BP1:1118 & BP $2: 425$ & 1.4 \\
\hline \multirow{2}{*}{ cfa-miR-219-3p } & CFDP1:10445 & CFDP2:2774 & 1.91 \\
\hline & BP1:1118 & CFDP1:10445 & 3.22 \\
\hline \multirow{3}{*}{ cfa-miR-138b } & BP2:425 & CFDP2:2774 & 2.71 \\
\hline & CFDP1:8716 & CFDP2:1414 & 2.62 \\
\hline & BP1:1571 & CFDP1:8716 & 2.47 \\
\hline \multirow{2}{*}{ cfa-miR-202 } & CFDP $1: 7366$ & CFDP2:1598 & 2.2 \\
\hline & BP1:1398 & CFDP1:7366 & 2.4 \\
\hline \multirow{2}{*}{ cfa-miR-449 } & BP1:1917 & BP2:4810 & 1.33 \\
\hline & BP2:4810 & CFDP2:1484 & 1.7 \\
\hline--------- & BP $1: 1651$ & BP2:615 & 1.42 \\
\hline \multirow[t]{2}{*}{ cfa-miR-139 } & CFDP1:4883 & CFDP2?:1124 & 2.12 \\
\hline & BP1:1651 & CFDP $1: 4883$ & 1.56 \\
\hline \multirow{2}{*}{ cfa-miR-448 } & BP1:1904 & BP2:693 & 1.46 \\
\hline & CFDP $1: 2645$ & CFDP2:919 & 1.53 \\
\hline \multirow{2}{*}{ cfa-miR-224 } & $\mathrm{BP} 1: 626$ & BP2:1378 & 1.14 \\
\hline & BP1:626 & CFDP1:2231 & 1.83 \\
\hline \multirow{2}{*}{ cfa-miR-215 } & CFDP1:597 & CFDP2:1940 & 1.7 \\
\hline & BP2:754 & CFDP2:1940 & 1.36 \\
\hline--------- & CFDP $1: 115$ & CFDP $2: 269$ & 1.22 \\
\hline \multirow[t]{2}{*}{ cfa-miR-34b } & BP1:1331 & CFDP1:115 & 3.53 \\
\hline & BP2:1907 & CFDP2:269 & 2.82 \\
\hline \multirow{2}{*}{ cfa-miR-1 } & CFDP 1:1505 & CFDP2:687 & 1.13 \\
\hline & BP 1:439 & BP2:1505 & 1.78 \\
\hline \multirow{2}{*}{ cfa-miR-1307 } & BP1:306 & BP2:875 & 1.51 \\
\hline & BP2:355 & CFDP2:1375 & 1.95 \\
\hline \multirow{4}{*}{ cfa-miR-802 } & BP1:812 & BP2:347 & 1.23 \\
\hline & CFDP1:115 & CFDP2:1086 & 3.24 \\
\hline & BP1:812 & CFDP1:115 & 2.82 \\
\hline & BP2:347 & CFDP2:1086 & 1.65 \\
\hline
\end{tabular}




\begin{tabular}{|c|c|c|c|}
\hline \multirow{4}{*}{$\begin{array}{l}\text { cfa-miR-346 } \\
\text { cfa-miR-1343 }\end{array}$} & CFDP1:1329 & CFDP2:234 & 2.51 \\
\hline & BP1:200 & CFDP1:1329 & 2.73 \\
\hline & BP1:8219 & CFDP1:22031 & 1.42 \\
\hline & CFDP 1:22031 & CFDP2:10113 & 1.12 \\
\hline \multirow{2}{*}{ cfa-miR-769 } & BP1:2087 & CFDP1:4473 & 1.1 \\
\hline & CFDP 1:4473 & CFDP2:1855 & 1.27 \\
\hline \multirow{2}{*}{ cfa-miR-8903 } & BP1:243 & CFDP1:815 & $1 . \overline{75}$ \\
\hline & CFDP1:815 & CFDP2:264 & 1.62 \\
\hline \multirow{2}{*}{ cfa-miR-8908a-3p } & BP 1:4530 & BP2:1269 & 1.84 \\
\hline & BP2:1269 & CFDP2:2759 & 1.12 \\
\hline \multirow{2}{*}{ cfa-miR-8908a-5p } & BP $1: 1375$ & BP 2:349 & 1.98 \\
\hline & BP $1: 1375$ & CFDP1:515 & 1.42 \\
\hline \multirow{3}{*}{ cfa-miR-8908b } & BP 1:1165 & BP2:264 & 2.14 \\
\hline & $\mathrm{BP} 1: 1165$ & CFDP1:565 & 1.04 \\
\hline & BP1:1650 & $\mathrm{BP} 2: \overline{2}$ & 2.53 \\
\hline \multirow[t]{2}{*}{ cfa-miR-8908d } & BP1:1650 & CFDF & 1.05 \\
\hline & BP2:286 & CFDP2:943 & 1.72 \\
\hline \multirow{2}{*}{ cfa-miR-34a } & $\mathrm{BAC}$ & $\mathrm{CFD}$ & 1.5 \\
\hline & BAC1:84320 & CFDAC1:197301 & 1.23 \\
\hline \multirow{2}{*}{ cfa-miR-338 } & BAC $1: 62453$ & $\mathrm{BAC} 2$ & 1.43 \\
\hline & BAC2:168721 & CFDA & 1.08 \\
\hline \multirow{2}{*}{ cfa-miR-758 } & $\mathrm{BAC} 2: 78102$ & CFDAC2:33774. & 1.21 \\
\hline & BAC1:61499 & CFDAC1:25712 & 1.26 \\
\hline \multirow{2}{*}{ cfa-miR-9 } & $\mathrm{BAC} 1: 24395$ & BAC2:68265.21 & 1.48 \\
\hline & BAC2:68265 & CFDAC2:27267.15 & 1.33 \\
\hline \multirow{2}{*}{ cfa-miR-138a } & BAC1:10468 & BAC2:35790 & 1.77 \\
\hline & BAC2:35790 & CFDAC2:7959 & 2.17 \\
\hline \multirow{2}{*}{ cfa-miR-124 } & $\mathrm{BAC} 1: 6200$ & BAC2:24560 & 1.99 \\
\hline & BAC2:24560 & CFDAC2:5837 & 2.07 \\
\hline \multirow{3}{*}{ cfa-miR-122 } & $\mathrm{BAC} 1: 13068$ & BAC2:591 & 4.47 \\
\hline & CFDAC1:16590 & CFDAC $2: 538$ & 4.95 \\
\hline & $\mathrm{BAC} 1: 10230$ & BAC2:1931 & 2.41 \\
\hline \multirow[t]{2}{*}{ cfa-miR-196b } & BAC2:1931 & CFDAC2:4345 & 1.17 \\
\hline & BAC1:10230 & CFDAC1:2254 & 2.18 \\
\hline \multirow{2}{*}{ cfa-miR-592 } & CFDAC $1: 2031$ & CFDAC2:4893 & 1.27 \\
\hline & BAC1:4316 & CFDAC1:2031 & 1.09 \\
\hline \multirow{2}{*}{ cfa-miR-31 } & $\mathrm{BAC} 1: 787$ & BAC2:2290 & 1.54 \\
\hline & $\mathrm{BAC} 1: 787$ & CFDAC1:1890 & 1.26 \\
\hline \multirow{2}{*}{ cfa-miR-138b } & BAC $1: 1216$ & BAC2:4243 & $\overline{1} . \overline{8}$ \\
\hline & BAC2:4243 & CFDAC2:985 & 2.11 \\
\hline
\end{tabular}




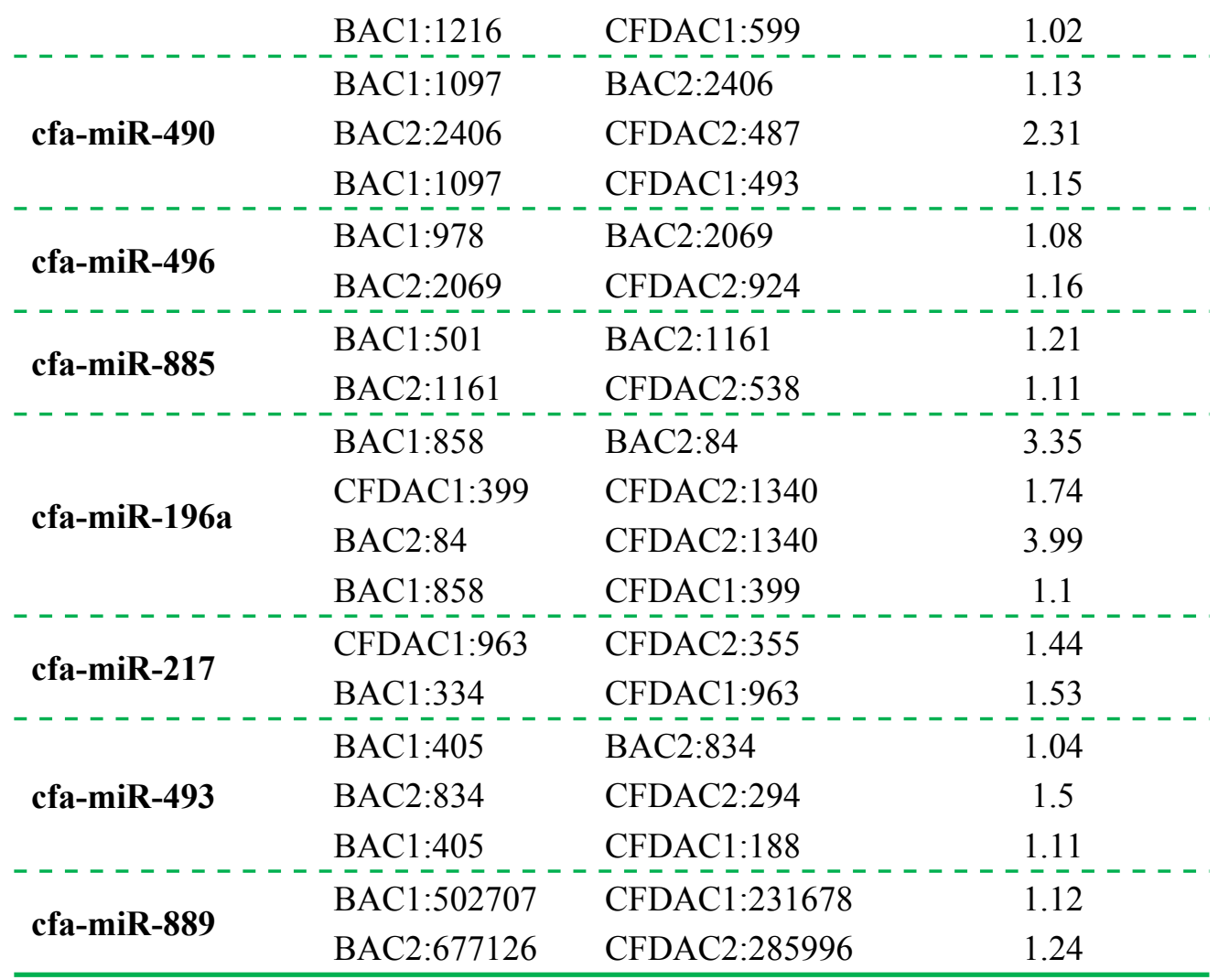

3 MiRNAs ranked up to down by the total reads number within adrenal cortex samples, with that the total reads

4 were greater than 1000. BAC1, Beagle adrenal cortex with treatment; BAC2, Beagle adrenal cortex without

5 treatment; BP1, Beagle pituitary with treatment; BP2, Beagle pituitary without treatment; CFDAC1, Chinese

6 Field Dog adrenal cortex with treatment; CFDAC2, Chinese Field Dog adrenal cortex without treatment;

7 CFDP1, Chinese Field Dog pituitary with treatment; CFDP2, Chinese Field Dog pituitary without treatment. 


\section{Table 3 (on next page)}

The potential differentially expressed miRNAs targeting the differentially expressed genes, without prejudice to a negative correlation between miRNA and its corresponding target genes in both breeds.

MiRNA and its corresponding potential target genes' expression level are indicated by normalized reads and FPKM (Fragments Per Kilobase of exon model per Million mapped reads) respectively. BP1, Beagle pituitary with treatment; BP2, Beagle pituitary without treatment; CFDP1, Chinese Field Dog pituitary with treatment; CFDP2, Chinese Field Dog pituitary without treatment. <?xml:namespace prefix = "o" ns = "urn:schemasmicrosoft-com:office:office" /> 
1 Table 3. The potential differentially expressed miRNAs targeting the differentially

2 expressed genes, without prejudice to a negative correlation between miRNA and its

3 corresponding target genes in both breeds.

4

\begin{tabular}{|c|c|c|c|c|}
\hline MiRNA \& Genes & BPC1 & $\mathrm{BPC} 2$ & TPC1 & ТРC2 \\
\hline cfa-miR-135b & 28942.56 & 15071.53 & 10065.27 & 21000.87 \\
\hline GRIN2B & 0.250209 & 0.327438 & 4.27929 & 0.252423 \\
\hline cfa-miR-205 & 10730.32 & 22724.29 & 12764.71 & 55847.22 \\
\hline CHN1 & 11.6623 & 8.20414 & 58.7752 & 9.90749 \\
\hline$G L R B$ & 5.47912 & 5.66417 & 30.3379 & 6.2008 \\
\hline$M M D$ & 14.1617 & 12.4959 & 41.8962 & 12.9108 \\
\hline cfa-miR-30a & 6285675 & 4436273 & 2553208 & 5289957 \\
\hline$S L C 1 A 2$ & 3.18748 & 4.03293 & 108.28 & 2.35374 \\
\hline$G R I N 2 A$ & 0.0143535 & 0.0892129 & 1.75419 & 0 \\
\hline$G R I A 2$ & 26.2931 & 26.7041 & 69.6217 & 19.3989 \\
\hline CAMTA1 & 4.61618 & 5.50413 & 14.7361 & 5.71324 \\
\hline SORCS3 & 12.1116 & 23.2745 & 12.4343 & 5.26901 \\
\hline$A T P 2 B 2$ & 2.74647 & 3.10767 & 18.8807 & 3.18689 \\
\hline$F A M 49 A$ & 7.91528 & 8.88205 & 39.4249 & 8.66222 \\
\hline cfa-miR-34b & 1331.3 & 1906.69 & 115.3 & 269.24 \\
\hline$N R X N 2$ & 3.15428 & 1.36425 & 24.2216 & 2.60845 \\
\hline$S C N 2 B$ & 2.91752 & 1.15905 & 42.2646 & 1.72229 \\
\hline SLC6A1 & 7.34403 & 2.86335 & 32.8953 & 6.54531 \\
\hline SYNPO & 4.77262 & 4.39135 & 21.2311 & 5.37488 \\
\hline GRM7 & 0.32311 & 0.330141 & 4.90747 & 0.391847 \\
\hline JAKMIP1 & 0.656131 & 0.477843 & 8.33219 & 0.783011 \\
\hline cfa-miR-802 & 812.1 & 346.67 & 115.3 & 1085.75 \\
\hline$N E C A B 1$ & 6.73288 & 4.95793 & 42.7074 & 7.71294 \\
\hline
\end{tabular}

5 MiRNA and its corresponding potential target genes' expression level are indicated by normalized reads and

6 FPKM (Fragments Per Kilobase of exon model per Million mapped reads) respectively.

7 BP1, Beagle pituitary with treatment; BP2, Beagle pituitary without treatment; CFDP1, Chinese Field Dog 8 pituitary with treatment; CFDP2, Chinese Field Dog pituitary without treatment. 


\section{Figure 1 (on next page)}

Length distribution of matched reads after quality control in adrenal cortex (A) and pituitary (B).

The white, light black, light green, and green columns represent the samples of pre-stress for Beagle, post-stress for Beagle, pre-stress for CFD, and post-stress for CFD, respectively. 

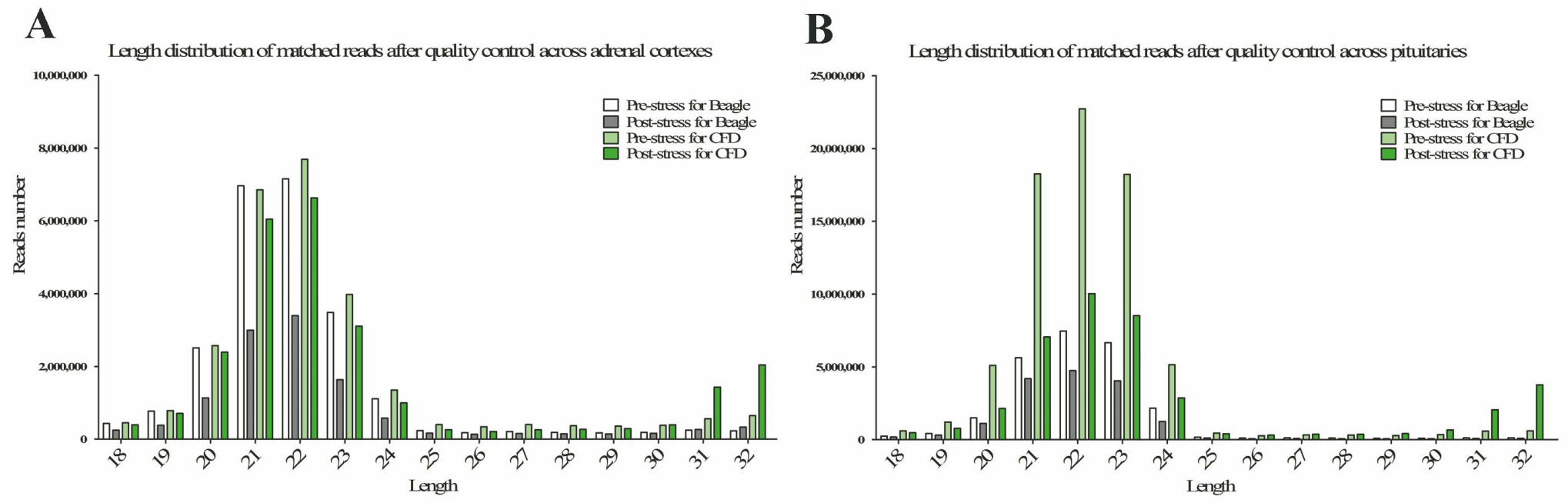
Figure 2 (on next page)

Distribution ration by small RNAs (SRNA) on genome. 
Distribution ratio on genome by sRNA

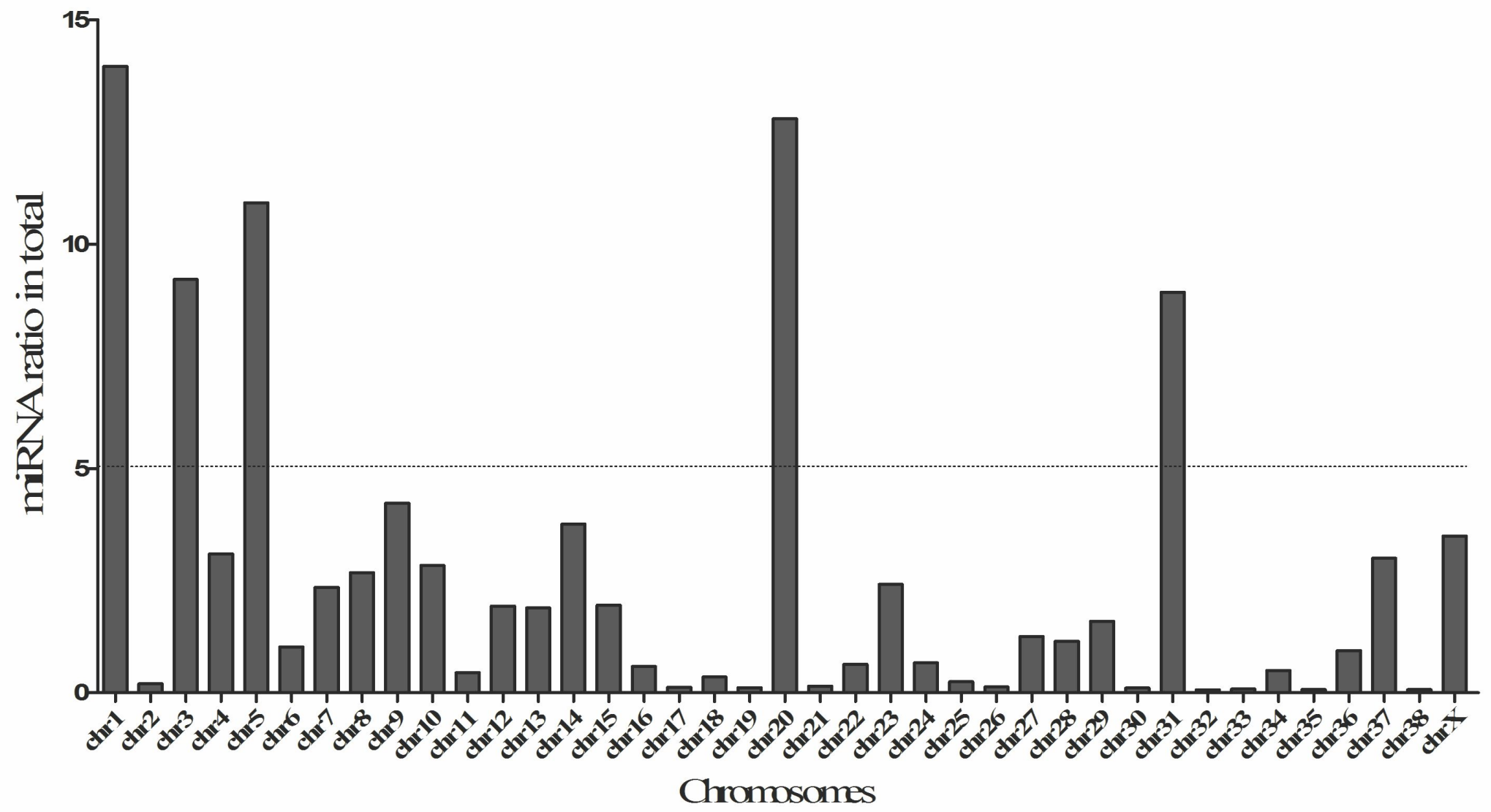


Figure 3 (on next page)

Venn diagrams of differentially expressed miRNA s (DE-miRNAs) among contrasts of each tissue.

A- T he DE-miRNA s distributed in pituitary, including BP1, BP2, CFDP1, and CFDP2. B- The DE -miRNA s distributed in adrenal cortex, including four groups of BAC1, BAC2, CFDAC1, and CFDAC2. The white number denotes the number of DEGs shared by at least two contrasts and are the ones selected for further analysis . 
A

CFDP1_vs_CFDP2 CFDP2_vs_BP2 BP1_vs_BP2

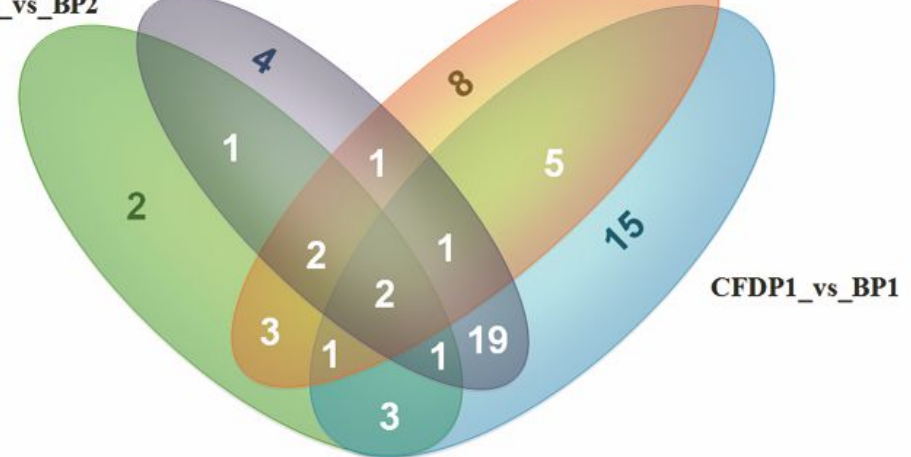

B CFDAC1_vs_CFDAC2 BAC1_vs_BAC2

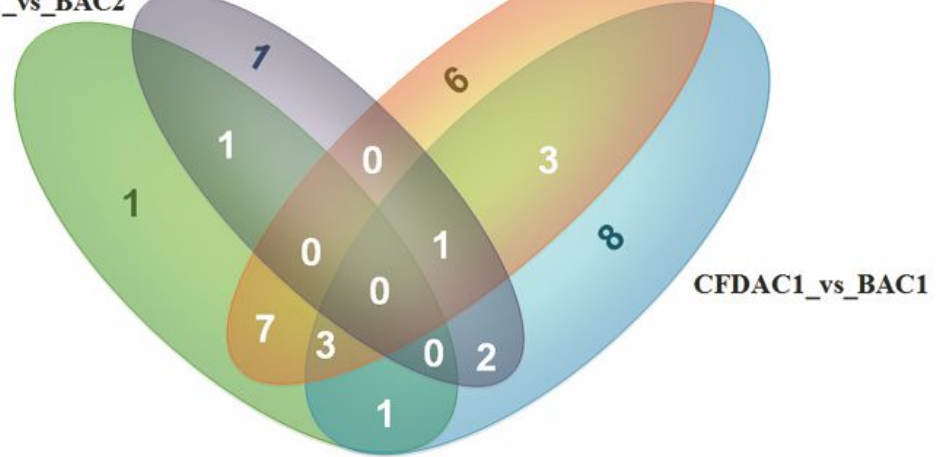




\section{Figure 4 (on next page)}

Validations of sRNA-seq by qPCR.

U6 and U48 are the internal control genes to normalize the expression level of cfa-miR-30a, cfa-miR-205, cfa-miR-124 and cfa-miR-222 in CFDP1_vs_CFDP2. CFDP1, CFD with chronic stress exposure; CFDP2, CFD without chronic stress exposure. 
sRNA-seq validated by qPCR in CFDP1_vs_CFDP2

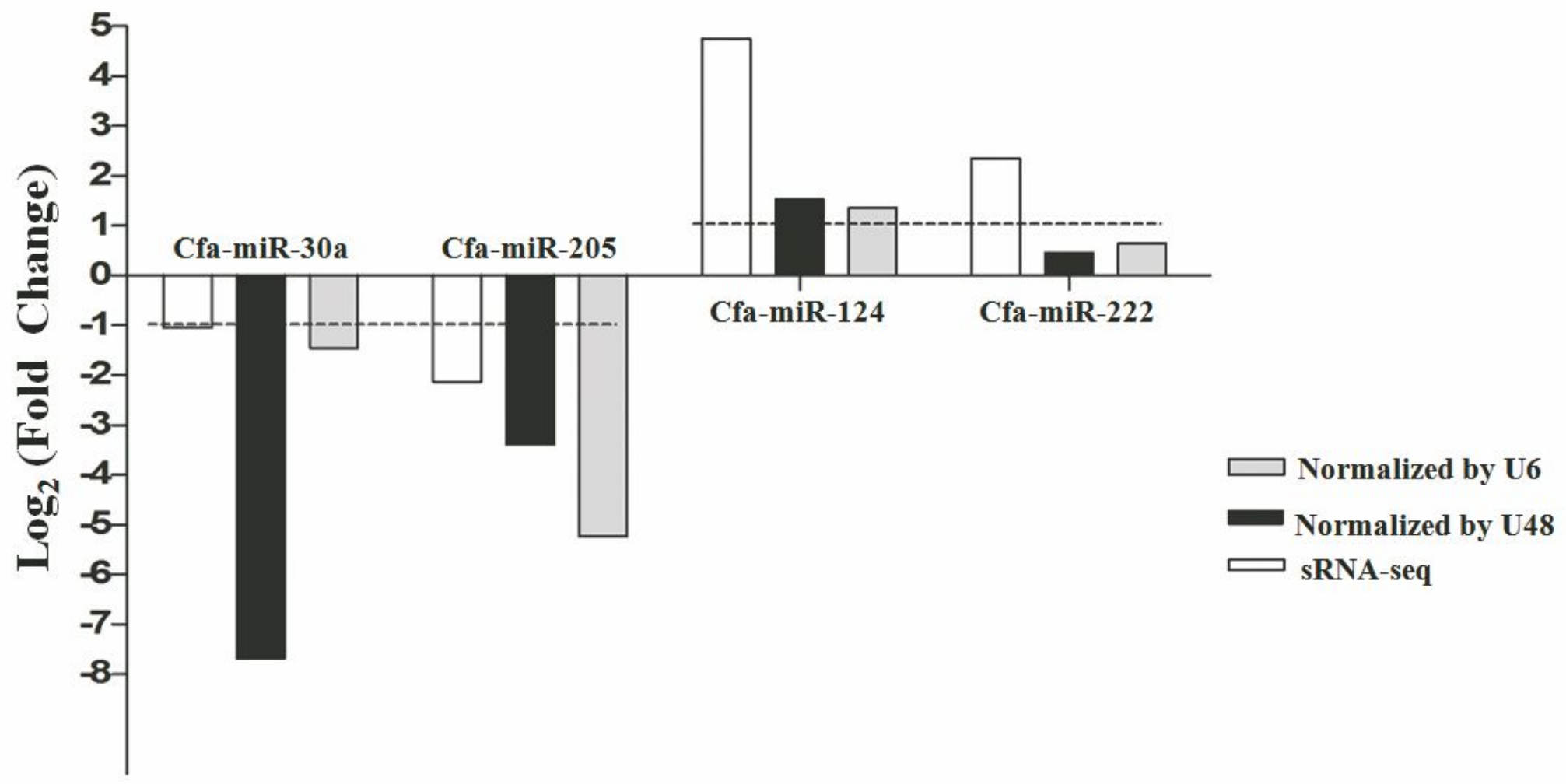


Figure 5 (on next page)

(A) Cfa-miR-205- MMD pair identification. (B) Cfa-miR-205 and MMD expression patterns in CFD hippocampus with and without chronic stress exposure. 
$\mathbf{A}$

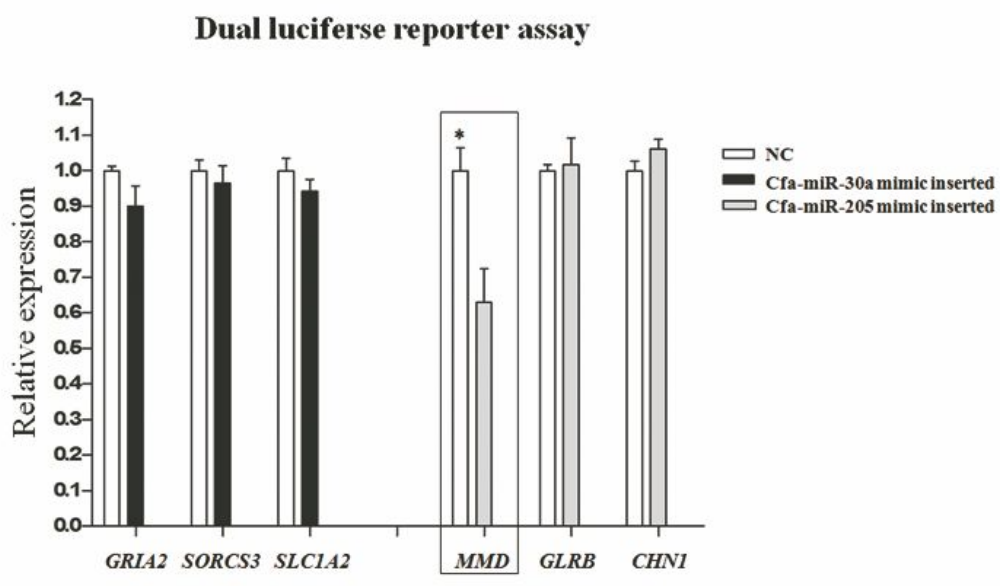

B

$M M D$ and cfa-miR-205 relative expression with and without stress exposure in CFD hippocampus

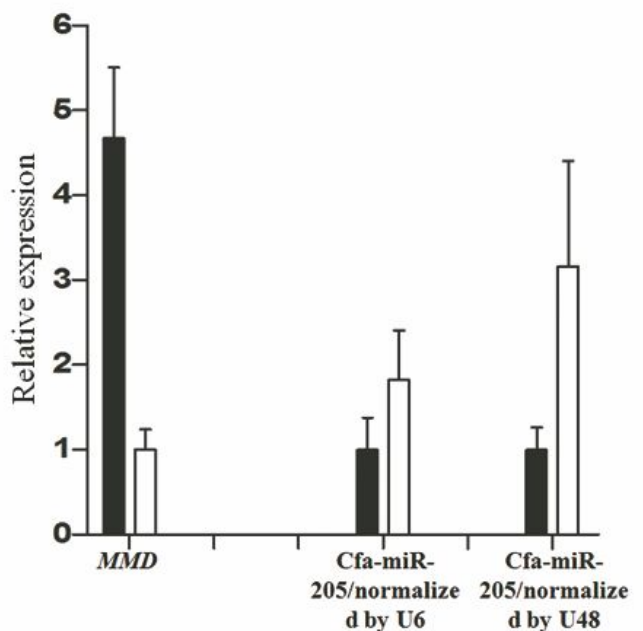

